\title{
Brachytrupes Algorithm for Solving Optimal Reactive Power Problem
}

\author{
Kanagasabai Lenin
}

Department of EEE, Prasad V.Potluri Siddhartha Institute of Technology, Kanuru, Vijayawada, Andhra Pradesh 520007, India

Corresponding Author Email: gklenin@gmail.com

https://doi.org/10.18280/isi.240105

Received: 10 November 2018

Accepted: 23 January 2019

\section{Keywords:}

optimal reactive power, real power, transmission loss, Brachytrupes

\begin{abstract}
In this work Brachytrupes Algorithm (BA) has been utilized to solve the optimal reactive power flow problem. Brachytrupes are predominantly cylindrical, and vertically flattened one. Its head is sphere-shaped with extended slender antennae arise from cone-shaped scapes. Pronotum is trapezoidal in shape, vigorous, and well-sclerotinized. A pair of long cerci is present at the tip of the abdomen. Ovipositor shape is cylindrical, long, narrow, smooth and shiny in the female brachytrupes. Back pair of legs generally known as the femora are significantly enlarged for jumping. A number of moveable spurs are there in the hind legs. One or more tympani are present in the tibiae of the front legs which are used for the reception of sound. Projected algorithm presumes that the probability of a Brachytrupes sound for resentment is $\mathrm{p}$ which is between 0 and 1. Once a Brachytrupes makes sound for resentment, it is whispered that they randomly walk to another male Brachytrupes for brawl. The convincing Brachytrupes takes consign of the solution and eradicate the loser Brachytrupes. Female Brachytrupes are seduced by male Brachytrupes sound for mating while remaining male Brachytrupes will move away. Brachytrupes will mate and generate offspring. They progress to an innovative place, which means they are taken to enhanced location in the search space. Proposed Brachytrupes Algorithm (BA) has been validated in standard IEEE 57, 300 test systems. Real Power loss has been reduced when compared to other standard reported algorithms.
\end{abstract}

\section{INTRODUCTION}

Reactive power optimization problem plays key role in protected \& profitable operation of the power system. Numerous conventional methods [1-6] have been used already for solving the problem. A variety of drawbacks have been found in the conventional methods and mainly difficulty in handling the inequality constraints. Last two decades many evolutionary algorithms [7-14]. In this work Brachytrupes Algorithm (BA) has been utilized to solve the optimal reactive power flow problem. Brachytrupes are predominantly cylindrical, and vertically flattened one. Its head is sphere-shaped with extended slender antennae arise from cone-shaped scapes. Pronotum is trapezoidal in shape, vigorous, and well-sclerotinized. A pair of long cerci is present at the tip of the abdomen. Ovipositor shape is cylindrical, long, narrow, smooth and shiny in the female brachytrupes. Back pair of legs generally known as the femora are significantly enlarged for jumping. A number of moveable spurs are there in the hind legs. One or more tympani are present in the tibiae of the front legs which are used for the reception of sound. On the body of Brachytrupes the wings are lie in flat mode and the fore wings are elytra made of tough chitin, stand-in as a protective guard for the soft parts of the body. In male Brachytrupes, stridulatory organ are present for the making of sound. Frequency and velocity of the sound is calculated. Secondly, when one Brachytrupes make sound for resentment other male Brachytrupes are beguile and other female Brachytrupes will move away. But All Brachytrupes will not make sound for resentment. Projected algorithm presumes that the probability of a Brachytrupes sound for resentment is $p$ which is between 0 and 1. Once a Brachytrupes makes sound for resentment, it is whispered that they randomly walk to another male Brachytrupes for brawl. The convincing Brachytrupes takes consign of the solution and eradicate the loser Brachytrupes. Female Brachytrupes are seduced by male Brachytrupes sound for mating while remaining male Brachytrupes will move away. Brachytrupes will mate and generate offspring. They progress to an innovative place, which means they are taken to enhanced location in the search space. Proposed Brachytrupes Algorithm (BA) has been validated in standard IEEE 57, 300 test systems. Real Power loss has been reduced when compared to other standard reported algorithms.

\section{PROBLEM FORMULATION}

Objective of the problem is to reduce the true power loss:

$\mathbf{F}=\mathbf{P}_{\mathrm{L}}=\sum_{\mathrm{k} \in \mathrm{Nbr}} \mathbf{g}_{\mathrm{k}}\left(\mathbf{V}_{\mathrm{i}}^{2}+\mathbf{V}_{\mathrm{j}}^{2}-2 \mathbf{V}_{\mathrm{i}} \mathbf{V}_{\mathrm{j}} \cos \boldsymbol{\theta}_{\mathrm{ij}}\right)$

Voltage deviation given as follows:

$F=P_{L}+\omega_{v} \times$ Voltage Deviation

Voltage deviation given by:

Voltage Deviation $\quad=\sum_{i=1}^{N p q}\left|V_{i}-1\right|$ 
Constraint (Equality)

$\mathbf{P}_{\mathbf{G}}=\mathbf{P}_{\mathrm{D}}+\mathbf{P}_{\mathbf{L}}$

Constraints (Inequality)

$\mathbf{P}_{\text {gslack }}^{\min } \leq \mathbf{P}_{\text {gslack }} \leq \mathbf{P}_{\text {gslack }}^{\max }$

$\mathbf{Q}_{\mathrm{gi}}^{\mathrm{min}} \leq \mathbf{Q}_{\mathrm{gi}} \leq \mathbf{Q}_{\mathrm{gi}}^{\mathrm{max}}, \mathbf{i} \in \mathbf{N}_{\mathrm{g}}$

$\mathbf{V}_{\mathbf{i}}^{\min } \leq \mathbf{V}_{\mathbf{i}} \leq \mathbf{V}_{\mathbf{i}}^{\max }, \mathbf{i} \in \mathbf{N}$

$\mathrm{T}_{\mathbf{i}}^{\min } \leq \mathrm{T}_{\mathbf{i}} \leq \mathrm{T}_{\mathrm{i}}^{\max }, \mathbf{i} \in \mathrm{N}_{\mathrm{T}}$

$\mathrm{Q}_{\mathrm{c}}^{\min } \leq \mathrm{Q}_{\mathrm{c}} \leq \mathrm{Q}_{\mathrm{C}}^{\max }, \mathrm{i} \in \mathrm{N}_{\mathrm{C}}$

\section{BRACHYTRUPES ALGORITHM}

Brachytrupes are predominantly cylindrical, and vertically flattened one. Its head is sphere-shaped with extended slender antennae arise from cone-shaped scapes. Pronotum is trapezoidal in shape, vigorous, and well-sclerotinized.

A pair of long cerci is present at the tip of the abdomen. Ovipositor shape is cylindrical, long, narrow, smooth and shiny in the female Brachytrupes. Back pair of legs generally known as the femora are significantly enlarged for jumping. A number of moveable spurs are there in the hind legs. One or more tympani are present in the tibiae of the front legs which are used for the reception of sound.

On the body of Brachytrupes the wings are lie in flat mode and the fore wings are elytra made of tough chitin, stand-in as a protective guard for the soft parts of the body. In male Brachytrupes, stridulatory organ are present for the making of sound. Frequency and velocity of the sound is calculated. Secondly, when one Brachytrupe make sound for resentment other male Brachytrupes are beguile and other female Brachytrupes will move away. But All Brachytrupes will not make sound for resentment. Projected algorithm presumes that the probability of a Brachytrupes sound for resentment is $\mathrm{p}$ which is between 0 and 1 . Once a Brachytrupes makes sound for resentment, it is whispered that they randomly walk to another male Brachytrupes for brawl. The convincing Brachytrupes takes consign of the solution and eradicate the loser Brachytrupes. Female Brachytrupes are seduced by male Brachytrupes sound for mating while remaining male Brachytrupes will move away. Brachytrupes will mate and generate offspring. They progress to an innovative place, which means they are taken to enhanced location in the search space.

\subsection{Calculation of sound rate}

The rate at which Brachytrupes makes sound is depend on relationship of air and temperature

Number of sound per minute $\left(\mathrm{N}_{\mathrm{S}}\right)$ is calculated with temperature $\left(\mathrm{T}_{\mathrm{S}}\right)$

$T_{s}=49.80+\frac{N_{c}-38.90}{3.85}$

Number of sound per minute $\left(\mathrm{N}_{\mathrm{S}}\right)$ is calculated with temperature $\left(\mathrm{T}_{\mathrm{S}}\right)$ in Celsius $\left(\mathrm{T}_{\mathrm{cs}}\right)$, is given by,

$T_{C S}=9.76+\frac{N_{c}-38.90}{6.89}$
At certain temperature $T_{c}$ and $T_{f}$, the sound rate is given by,

$N_{s}=\left(T_{c s}-9.76\right) * 6.89+38.90$

$N_{s}=\left(T_{s}-49.80\right) * 3.95+38.90$

Brachytrupes sound is computed based on the sound rate $\mathrm{N}_{\mathrm{s}}$, with respect to frequency $\mathrm{F}$ and velocity $\mathrm{V}$

$F_{c s}=N_{s} * \gamma$

$V l=F r * \lambda$

Each Brachytrupes step size of is computed as follows:

distance $=\frac{V l}{F r}$

Step size $=\beta *$ distance $*($ location - Best location $)$

New-fangled position of the Brachytrupes is calculated by,

New_fangled position $=$ location + step size $* \gamma$

Step 1

By using equations; $T_{s}=49.80+\frac{N_{c}-38.90}{3.85}$ or $T_{C S}=$ $9.76+\frac{N_{c}-38.90}{6.89}$ sound rate of each Brachytrupes will be computed.

By using equations; $F_{c s}=N_{s} * \gamma \quad$ and $\quad V l=F r * \lambda$ Brachytrupes frequency, velocity will be computed.

By using equations; distance $=\frac{V l}{F r}$ and Step size $=\beta$ * distance $*$ (location - Best location) step size will be computed.

By using the equation New_fangled position = location + step size $* \gamma$ Brachytrupes new positions will be updated.

Revisit of Brachytrupes towards novel locations End

\section{Step 2}

With respect to new location Male Brachytrupes randomly will pick a female Brachytrupes

A incise point will be chosen for the above procedure

Similar to a crossover in Genetic algorithm; genetic materials of both male and female Brachytrupes will be intermingled with reference to their incise point to generate two new-fangled offspring's.

Fitness of the offspring will be computed

Movement towards the new locations by the two offspring's and the parents Brachytrupes

End

\section{Step 3}

Brachytrupes will walk to new locations when random $>\mathrm{P}$

Fitness value of the Brachytrupes will be calculated in accordance to new locations

Best Brachytrupes will be chosen for further procedure

Movement towards the new locations by the Brachytrupes End 


\subsection{Main procedure}

Brachytrupes position will be located

Female Brachytrupes has to be chosen

Fitness value of each Brachytrupes has to be calculated

With respect to position choose the most excellent

Brachytrupes $\mathrm{f}_{\text {best_Brachytrupes }}$

Set gbest_Brachytrupes as the present $f_{\text {best_Brachytrupes }}$ and in the preliminary generation $g_{\text {best_Brachytrupes }}-f_{\text {best_Brachytrupes }}$

While (stop criteria is not met)

Then

Sound for mating - (step1)

Male Brachytrupes to mate with female Brachytrupes (step2)

Sound for resentment with probability $\mathrm{P}-($ step3)

Fitness value will be calculated

From the innovative positions choose the $\mathrm{f}_{\text {best_Brachytrupes, }}$
Modify $g_{\text {best_Brachytrupes }}$ with the present $\mathrm{f}_{\text {best_Brachytrupes. When }}$ $f_{\text {best_Brachytrupes }}>\mathrm{g}_{\text {best_B Brachytrupes, }}$,

End while

Revisit to comprehensive best Brachytrupes at cessation End

\section{SIMULATION RESULTS}

Performance of the proposed Brachytrupes Algorithm (BA) has been validated by tested in standard IEEE 57 bus system [15]. Total active and reactive power demands in the system are 1248.23 MW and 334.16 MVAR. Generator data the system is given in Table 1. The optimum loss comparison is presented in Table 2. Figure 1 gives the comparison of active power loss.

Table 1. Generator data

\begin{tabular}{|c|c|c|c|c|}
\hline Generator No & Pgi minimum & Pgi maximum & Qgi minimum & Qgi maximum \\
\hline 1 & 25.00 & 50.00 & 0.00 & 0.00 \\
\hline 2 & 15.00 & 90.00 & -17.00 & 50.00 \\
\hline 3 & 10.00 & 500.00 & -10.00 & 60.00 \\
\hline 4 & 10.00 & 50.00 & -8.00 & 25.00 \\
\hline 5 & 12.00 & 50.00 & -140.00 & 200.00 \\
\hline 6 & 10.00 & 360.00 & -3.00 & 9.00 \\
\hline 7 & 50.00 & 550.00 & -50.00 & 155.00 \\
\hline
\end{tabular}

Table 2. Comparison of losses

\begin{tabular}{|c|c|c|c|c|c|c|c|c|}
\hline Parameter & $\begin{array}{c}\text { CLPSO } \\
{[17]}\end{array}$ & $\begin{array}{c}\text { DE } \\
{[16]}\end{array}$ & $\begin{array}{l}\text { GSA } \\
{[16]}\end{array}$ & $\begin{array}{c}\text { OGSA } \\
{[18]}\end{array}$ & $\begin{array}{l}\text { SOA } \\
{[17]}\end{array}$ & $\begin{array}{c}\text { QODE } \\
{[16]}\end{array}$ & $\begin{array}{l}\text { CSA } \\
{[19]}\end{array}$ & $\overline{\mathbf{B A}}$ \\
\hline PLOSS (MW) & 24.5152 & 16.7857 & 23.4611 & 23.43 & 24.2654 & 15.8473 & 15.5149 & 14.0412 \\
\hline
\end{tabular}

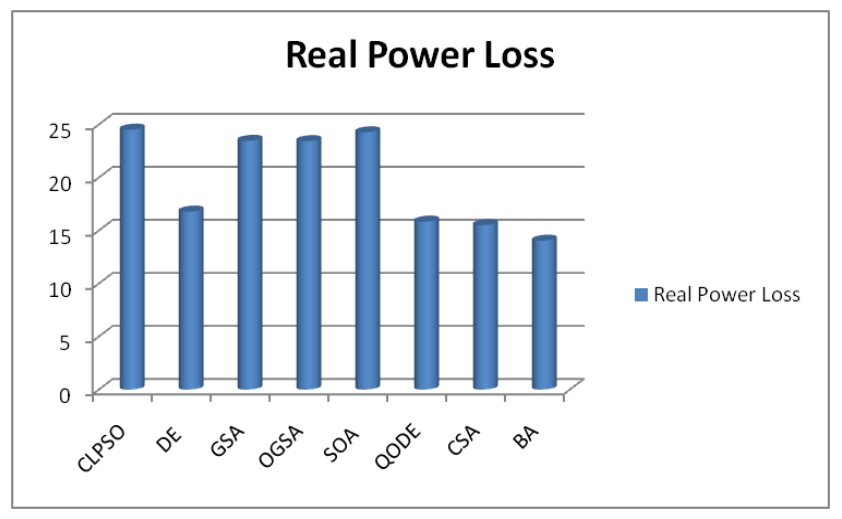

Figure 1. Comparison of active power loss

Then the performance of the proposed Brachytrupes Algorithm (BA) has been tested in standard IEEE 300 bus system [15]. Table 3 shows the comparison of real power loss obtained after optimization. Figure 2 gives the comparison of real power loss.

Table 3. Comparison of real power loss

\begin{tabular}{|c|c|c|c|c|}
\hline Parameter & EGA [20] & EEA [20] & CSA [19] & BA \\
\hline $\begin{array}{c}\text { PLOSS } \\
\text { (MW) }\end{array}$ & 646.2998 & 650.6027 & 635.8942 & 625.9864 \\
\hline
\end{tabular}

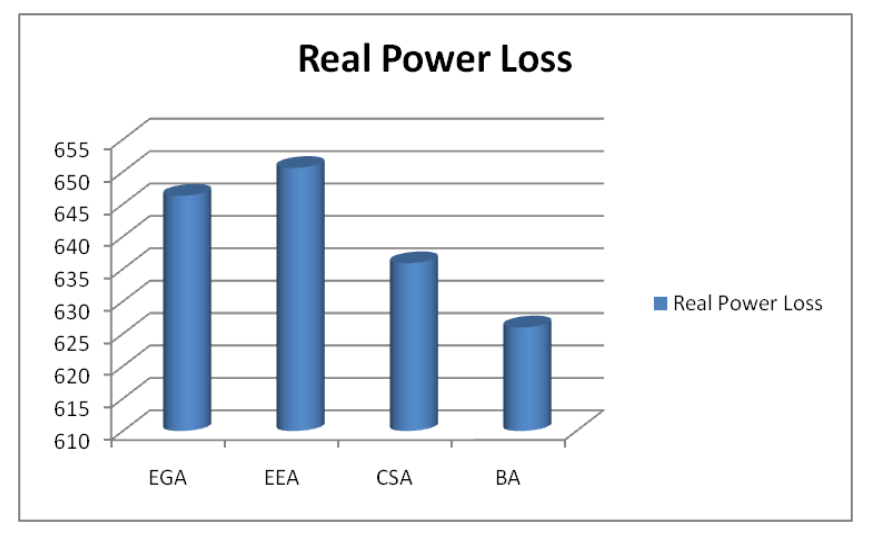

Figure 2. Real power loss comparison

\section{CONCLUSION}

In this paper Brachytrupes Algorithm (BA) successfully solved the optimal reactive power problem. Projected algorithm presumes that the probability of a Brachytrupes sound for resentment is $p$ which is between 0 and 1 . The convincing Brachytrupes takes consign of the solution and eradicate the loser Brachytrupes. Female Brachytrupes are seduced by male Brachytrupes sound for mating while remaining male Brachytrupes will move away. Brachytrupes will mate and generate offspring. They progress to an innovative place, which means they are taken to enhanced location in the search space. Proposed Brachytrupes 
Algorithm (BA) has been validated in standard IEEE 57, 300 test systems. Real Power loss has been reduced when compared to other standard reported algorithms.

\section{REFERENCES}

[1] Lee KY. (1984). Fuel-cost minimisation for both real andreactive-power dispatches. Proceedings Generation, Transmission and Distribution Conference 131(3): 8593.

[2] Deeb NI. (1998). An efficient technique for reactive power dispatch using a revised linear programming approach, Electric Power System Research 15(2): 121134. https://doi.org/10.1016/0378-7796(88)90016-8

[3] Bjelogrlic MR, Calovic MS, Babic BS. (1990). Application of Newton's optimal power flow in voltage/reactive power control. IEEE Trans Power System 5(4): 1447-1454.

[4] Granville S. (1994). Optimal reactive dispatch through interior point methods. IEEE Transactions on Power System 9(1): 136-146. http://dx.doi.org/10.1109/59.317548

[5] Grudinin N. (1998). Reactive power optimization using successive quadratic programming method. IEEE Transactions on Power System 13(4): 1219-1225. http://dx.doi.org/10.1109/59.736232

[6] Yan W, Yu J, Yu DC, Bhattarai K. (2006). A new optimal reactive power flow model in rectangular form and its solution by predictor corrector primal dual interior point method. IEEE Trans. Pwr. Syst 21(1): 6167. http://dx.doi.org/10.1109/TPWRS.2005.861978

[7] Aparajita M, Vivekananda M. (2015). Solution of optimal reactive power dispatch by chaotic krill herd algorithm. IET Generation, Transmission, Distribution, 9(15): 2351-2362. http://dx.doi.org/10.1049/ietgtd.2015.0077

[8] Hu Z, Wang X. (2010). Stochastic optimal reactive power dispatch: Formulation and solution method. Electric Power Energy Syst 32: 615-621. http://dx.doi.org/10.1016/j.ijepes.2009.11.018

[9] Morgan MAP, Abdullah RH, Sulaiman MH, Mustafa M, Samad R. (2016). Multi-objective evolutionary programming (MOEP) using mutation based on adaptive mutation operator (AMO) applied for optimal reactive power dispatch. ARPN Journal of Engineering and Applied Sciences 11(14): 1-20.
[10] Pandiarajan K, Babulal CK. (2016). Fuzzy harmony search algorithm based optimal power flow for power system security enhancement. International Journal Electric Power Energy Syst 78: 72-79. https://doi.org/10.1016/j.ijepes.2015.11.053

[11] Morgan M, Abdullah NRH, Sulaiman MH, Mustafa M, Samad R. (2016). Benchmark Studies on optimal reactive power dispatch (ORPD) based multi-objective evolutionary programming (MOEP) using mutation based on adaptive mutation adapter (AMO) and polynomial mutation operator (PMO). Journal of Electrical Systems 12-21.

[12] Mei RGS, Sulaiman MH, Mustaffa Z. (2016). Ant lion optimizer for optimal reactive power dispatch solution. Journal of Electrical Systems, AMPE2015 68-74.

[13] Gagliano A, Nocera F. (2017). Analysis of the performances of electric energy storage in residential applications, International Journal of Heat and $\begin{array}{lll}\text { Technology } & 35(\mathrm{~S} 1) \text { : } & \text { S41-S48. }\end{array}$ https://doi.org/10.18280/ijht.35Sp0106

[14] Caldera M, Ungaro P, Cammarata G, Puglisi G. (2018). Survey-based analysis of the electrical energy demand in Italian households. Mathematical Modelling of Engineering Problems 5(3): 217-224. https://doi.org/10.18280/mmep.050313

[15] Power Systems Test Case Archive. Available. http://www2.ee.washington.edu/research/pstca/, accessed on November 11, 2018.

[16] Basu M. (2016). Quasi-oppositional differential evolution for optimal reactive power dispatch. Electrical Power and Energy Systems 78: 29-40. https://doi.org/10.1016/j.ijepes.2015.11.067

[17] Dai C. (2009). Seeker optimization algorithm for optimal reactive power dispatch. IEEE Trans. Power Systems 24(3): 1218-1231.

[18] Shaw B. (2014). Solution of reactive power dispatch of power systems by an opposition-based gravitational search algorithm. International Journal of Electrical Power Energy Systems 55: 29-40. https://doi.org/10.1016/j.ijepes.2013.08.010

[19] Reddy SS. (2017). Optimal reactive power scheduling using cuckoo search algorithm. International Journal of Electrical and Computer Engineering 7(5): 2349-2356.

[20] Reddy SS. (2014). Faster evolutionary algorithm based optimal power flow using incremental variables. Electrical Power and Energy Systems 54: 198-210. https://doi.org/10.1016/j.ijepes.2013.07.019 